\title{
Failure analysis of long round thread in horizontal well casing under multi-axis loading
}

\author{
Lin Tiejun $^{1}$, Li Zhaoyang ${ }^{1 *}$, Zhao Zhaoyang ${ }^{1}$, Bao Xiaomin ${ }^{1}$, Zhang Qiang ${ }^{1}$ \\ ${ }^{1}$ State Key Laboratory of Oil and Gas Reservoir Geology and Exploration, Southwest Petroleum University, Chengdu, Sichuan, 610500, \\ China
}

\begin{abstract}
The casing is subjected to complicated forces underground, and the threaded joint of the casing is the weak link of the casing. becoming more and more severe, various kinds of failure accidents often occur in practical use. Therefore, in view of the casing thread fracture failure during the process of volumetric fracturing in well $\mathrm{W}$ of an oil field. The finite element model of 5-1/2"API casing long round threaded joint was established in this paper, ABAQUS software was used to simulate and analyze the stress and deformation of casing thread under the loading state of overlock, axial tension and pressure, and fracturing internal pressure. The results show that the stress distribution of teeth is reasonable. Under the condition of axial tension and compression, the maximum stress of casing thread exceeds the yield strength into plasticity and causes damage. However, when fracturing and stimulation technology is implemented, the stress of the collar and casing body increases significantly, and the fracture is caused by fatigue and extended fracture under the alternating fracturing load. The finite element analysis results are consistent with the field failure results. Study the influence of downhole complex working condition on casing thread by simulation, which is of great significance to the protective casing.
\end{abstract}

\section{Introduction}

Fracturing and stimulation technology, as a conventional and very effective way to increase production, has been widely used in oil fields at home and abroad. However, with the application of fracturing and stimulation technology, problems such as leakage and wellbore stability occur. In the fracturing process, due to the high pressure, the connection between casing and collar is vulnerable to multiple loads such as bending and stretching. In addition, due to the influence of liquid corrosion and other factors in the well, the threaded joint at the casing connection is prone to fatigue fracture, which seriously affects the tightness and integrity of the casing connection. In case of serious cases, the string may even fall out of the well. As a result, the well is scrapped, causing huge economic losses.

Regarding the fracture failure of the threaded connection, zhang $\mathrm{xu}$ found that the casing material presented brittleness at low temperature, and local stress concentration occurred on the surface of the casing tube, which resulted in brittle fracture of the casing tube[1]. Zeng zhong proposed that non-standard operation in the upper shackle would lead to the increase of local contact stress in the thread segment and the increase of thread advance resistance, aiming at the fracture failure of steel casing of P110 steel grade [2]. Zhang jiyin found that API SOEC 5CT did not take into account the shoulder effect of special threaded connection, which made the coupling prone to cracking during the compaction of shale gas development Wells[3]. In 2008, zhu xiaohua proposed that both ends of the threaded joint are the starting point of fracture, and the contact pressure increases with the increase of axial tension[4]. Lian zhanghua found that the turbosha-connected threads tend to generate stress concentration in the first tooth under the action of bending and torsion load, which eventually leads to failure[5-6]. Liu yuan proposed that in a certain range of internal pressure, the connection strength of the joint has little relationship with the change of internal pressure, and the threaded joint may break and fail in a certain range of axial tension[7]. Chen ronghua proposed that the main reason of plastic deformation was the eccentricity error of the screw hole of the fixture, which made the local thread tooth of the fixture thread bear greater stress[8]. Zhang le proposed that the compression load would increase the thread sealing surface, the contact force of the shoulder and the contact length, but it would also increase the stress, and there was a failure risk[9]. Xu honglin proposed to adopt internal and external thickening of the end, avoid using special clearance collar for thick wall tubing, and reasonable operating torque on the shoulder of the console is conducive to improving the connection efficiency and strength[10].

The above scholars have conducted a detailed study on the causes and forms of failure of thread teeth and interference fit at thread joints. However, there are few references on the causes of thread failure during volumetric fracturing in ultra-deep horizontal Wells with large horizontal lengths and deep depths. In view of the

\footnotetext{
*Corresponding author's e-mail: 2697709648@qq.com
} 
above shortcomings, this paper mainly USES the finite element method to establish the finite element model of relevant casing threads according to the actual field conditions. At the same time, the stress form of the thread under the compound working condition was analyzed. Therefore, the corresponding conclusion can be drawn.

\section{Casing thread stress analysis model}

\subsection{Failure analysis of casing thread in horizontal well in block}

Well $\mathrm{W}$ of an oil field is a horizontal well with a practical depth of $5128.47 \mathrm{~m}$ and a vertical depth of $3099.72 \mathrm{~m}$. It has a set of cement back height of $1906 \mathrm{~m}$. The maximum well inclination Angle is $81.86^{\circ}$, the maximum dogleg degree is $7 \% / 30 \mathrm{~m}$, and the horizontal displacement is $2094 \mathrm{~m}$. The well deflected from $2420 \mathrm{~m}$ and twisted azimuth. The target well of horizontal well $\mathrm{A}$ is $2787.22 \mathrm{~m}$ deep, and the second hole is $5128.47 \mathrm{~m}$ deep. After fracturing the casing in well $\mathrm{W}$ at the 5 th stage, it was preliminarily judged to be a casing fracture on site. After putting forward the casing, it was found that the casing joint was fractured at the 2 nd and 3 rd joints. The casing fracture in the field is shown in figure 1 .

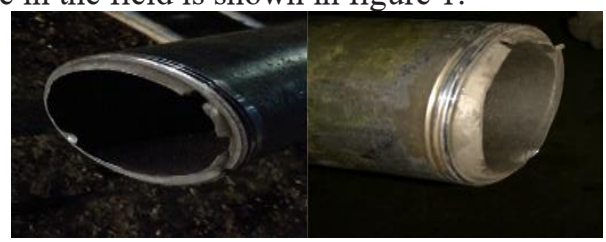

Figure 1. Schematic diagram of casing fracture in site

\subsection{Establish the finite element analysis model}

In this paper, based on the finite element software ABAQUS, a three-dimensional finite element analysis model is established by taking the $5-1 / 2$ "API long-round threaded joint as an example, as shown in figure 2 . The model parameters are shown in table 1.

Table $15-1 / 2$ " API casing long round thread parameters

\begin{tabular}{|c|c|}
\hline parameter & The numerical \\
\hline specifications & $5 \frac{1 / 2}{5}$ \\
\hline The big end diameter $/ \mathrm{mm}$ & 5.500 \\
\hline Number of threads per inch $/ \mathrm{mm}$ & 8 \\
\hline Length from tube end to hand tight surface /mm & 2.421 \\
\hline Effective thread length $/ \mathrm{mm}$ & 3.213 \\
\hline Total length from end to vanishing point $/ \mathrm{mm}$ & 3.500 \\
\hline Middle diameter of hand tight surface $/ \mathrm{mm}$ & 5.40337 \\
\hline Length from the end of the rear pipe to the center of the collar $/ \mathrm{mm}$ & 0.500 \\
\hline Length from end face of collar to hand tight face /mm & 0.704 \\
\hline Collar boring diameter $/ \mathrm{mm}$ & $5^{19 / 32}$ \\
\hline Depth of collar boring $/ \mathrm{mm}$ & 0.5500 \\
\hline Hands close to teeth number & 3 \\
\hline Minimum length of full top screw from tube end /mm & 2.375 \\
\hline
\end{tabular}

In this model, the casing steel grade is TP125 (minimum yield strength $862 \mathrm{MPa}$ ) and the wall thickness is $9.17 \mathrm{~mm}$; API long round thread joint $3 \mathrm{~d}$ structure, male and female thread face hand tight to machine tight contact interference top buckle; During the process of casing lowering or fracturing, the axial force $\mathrm{F}$ is $0 \sim 100 \mathrm{t}$ tension and $0 \sim 100 t$ compression force. The bending shear stress of the casing is applied to the joint under machine tightness, and $\mathrm{Q}$ was $0 \sim 100 \mathrm{MPa}$ in the $\mathrm{x}$ direction. The internal fracturing pressure of casing is applied to the joint in machine tight state, $\mathrm{P}$ is $0 \sim 100 \mathrm{MPa}$.

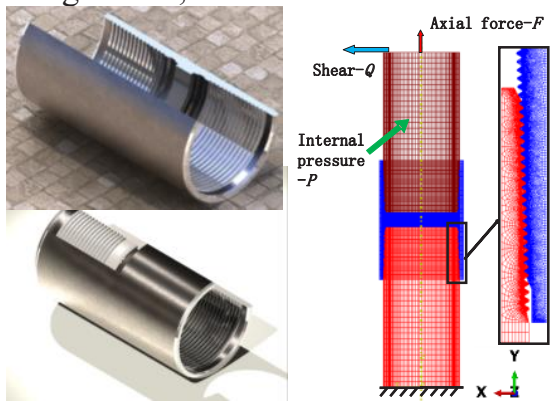

Figure 2. Three-dimensional structure and finite element model of long round thread

\section{Finite element analysis of stress and strain of casing thread}

Due to the complex borehole trajectory of $\mathrm{W}$ horizontal Wells, when the casing cannot be lowered smoothly, the field usually adopts the auxiliary method of lifting, flushing and releasing the casing to force the casing down, and at the same time, volume fracturing technology is used to achieve the purpose of effective stimulation. Therefore, based on the $51 / 2$ in casing long round thread finite element model established above, the stress change of casing joint thread under the condition of overlock, axial tension and fracturing internal pressure is studied according to the actual field conditions.

\subsection{Buckled state}

In the casing threaded joint model, the stress distribution of the threaded joint under the condition of top buckle is shown in figure 3 . The male and female joint rotates three buckles from hand tightening to machine tightening, and the tooth surface produces interference volume, and the 
tooth stress distribution is reasonable, and the root stress at both ends is the largest. The stress of the thread is mainly concentrated in the middle part. The stress of the lower common buckle gradually decreases from the top end to the bottom end. The stress reaches $827.2 \mathrm{MPa}$ at the top buckle. The stress of the lower half collar gradually increases from the upper end to the lower end, and the maximum stress is in the lower buckle, at which time the stress value is $944.8 \mathrm{MPa}$.

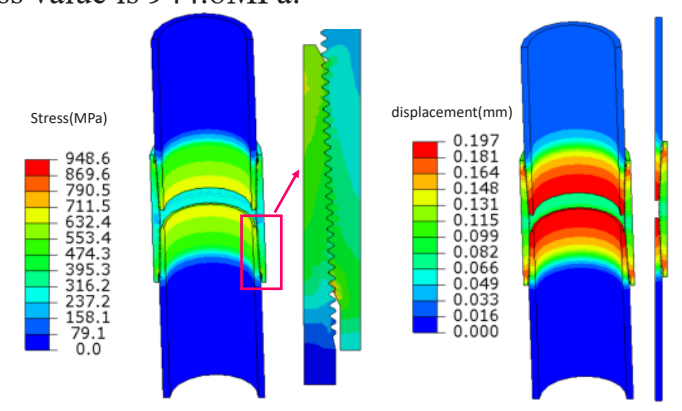

Figure 3. Stress - displacement distribution of long - round thread joint under top - buckle condition

Displacement distribution of threaded joint, interference of middle tooth surface is $0.13 \mathrm{~mm}$, interference displacement of tooth changes linearly, total displacement of joint transversely is $0.24 \mathrm{~mm}$. The contact force of the common buckle is evenly distributed in the middle, and the contact force at both ends is large, mainly in the first buckle at the upper end and the fifth buckle at the lower end, reaching $96.09 \mathrm{kN}$ at the maximum. The contact force is evenly distributed in the middle of the mother buckle, and the maximum contact force is mainly concentrated in the top fifth buckle and the bottom first buckle.

\subsection{Axial tension and pressure}

When the casing cannot be lowered smoothly, auxiliary methods are usually used to force the casing to be lowered in the field. In order to make clear the stress change of casing thread, the axial tension and pressure analysis of the measures adopted in the field were carried out.

(1) Axial tension

According to the lifting force on site, the simulated axial tension is $100 t$, and the stress distribution of the threaded joint under this working condition is shown in figure 4. Under the condition of machine tension, the maximum stress at the bottom of the 5-button tooth at the big end of the common buckle exceeds the elastic strength of the material (862MPa) and enters the plasticity, resulting in dangerous interface and damage. The stress distribution of the threaded joint is uniform, basically 423.4MPa. The stress in the lower part of the common buckle is small, the stress in the fourth buckle is the largest, the stress distribution in the lower part of the collar is relatively dispersed, and the maximum stress is in the lower buckle. However, the thread axial displacement of the casing joint is out of position, and the maximum displacement is $0.647 \mathrm{~mm}$. The last tooth in contact with the collar at the big end of the common buckle has the largest contact force, and the bearing capacity of the other teeth is even.

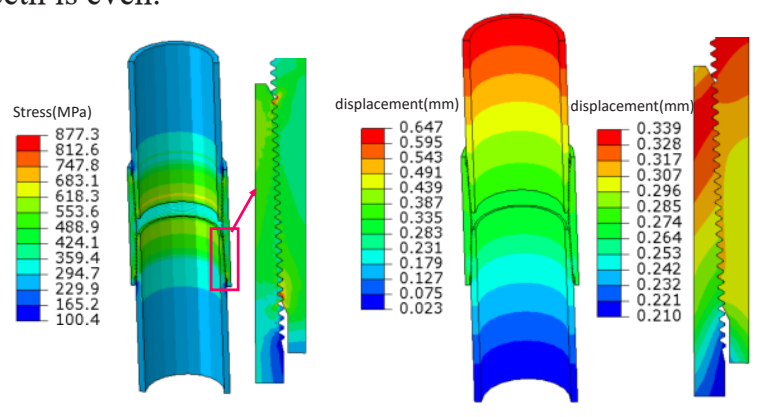

Figure 4. Stress-displacement distribution of long round threaded joints under $100 \mathrm{t}$ axial tension

(2) Axial pressure condition

The jacking and releasing of the casing is a multi-cycle operation process. For this reason, the axial force of the lower pressure is also 100t. The stress-displacement distribution of the threaded joint under this state is shown in figure 5. Under the condition of machine tightness, the axial compression is $100 \mathrm{t}$, and the maximum stress at the bottom of the fifth button-tooth of the big end of the common buckle and the cross-sectional hoop stress at the small end of the common buckle both enter into plasticity, and dangerous interface appears and damage occurs. The bottom stress of the fifth buckle at the end of the lower common buckle reaches the maximum, which is about 974.9 MPa. The stress of the lower half of the collar is relatively large, which reaches the maximum on the first buckle in contact with the lower common buckle. Joint thread axial displacement dislocation, the maximum displacement is $0.601 \mathrm{~mm}$. The contact force of the cross section of the big end and small end of the joint is the largest, and the bearing capacity of the other teeth is even.

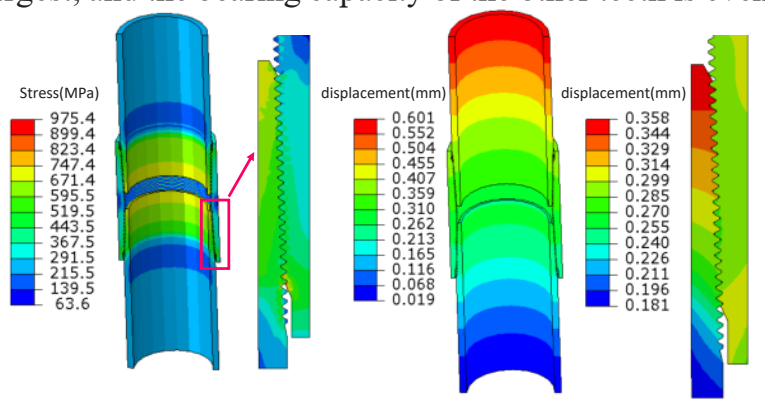

Figure 5. Stress-displacement distribution of long round threaded joints under $100 \mathrm{t}$ axial pressure

\subsection{Internal fracturing pressure}

When internal pressure of $46 \mathrm{MPa}$ is applied in the machine condition, the stress of the non-full top thread at the small end of the collar increases and produces plasticity, while the overall stress of the common thread decreases but the stress at the bottom and top of the teeth of the local thread at the large end increases to $865 \mathrm{MPa}$. When internal pressure of $100 \mathrm{MPa}$ is applied under the condition of machine tightness, the stress of the collar and casing body increases obviously, and the stress at the top and bottom of the meshing teeth of the common clasp increases, and more parts enter into plasticity. The stress of the lower common buckle decreases as a whole, and 
increases gradually from the lower end to the upper end. The stress of the fifth buckle at the lower end reaches the maximum, reaching $882.8 \mathrm{MPa}$.

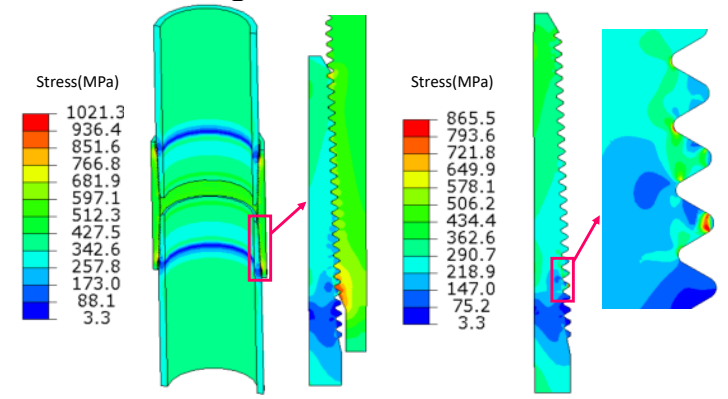

(a) Fracturing internal pressure is $46 \mathrm{Mpa}$

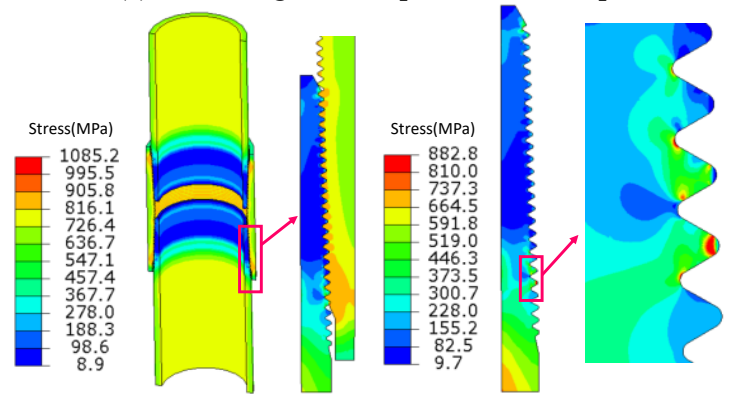

(b) Fracturing internal pressure is $100 \mathrm{Mpa}$

Figure 6. Stress distribution of long round thread joint and lower joint under fracturing internal pressure

By comparing the stress distribution of the long round threaded joint and the downlink under different internal pressure values in fracturing state in Figure 6, it can be seen that, due to the measures of lifting, punching and releasing in the process of casing lowering, as well as the obvious bending stress in the horizontal well with large inclination Angle, the dangerous interface and damage occurred at the threaded joint of casing. Under the above circumstances, different internal pressures were applied again, which resulted in the gradual increase of stress on the small end of the collar and the generation of plasticity, the gradual decrease of the overall stress on the common buckle, and the increase of stress on the top and bottom of the meshing teeth of the common buckle. The simulation results are consistent with the field conditions of casing failure due to fatigue initiation and extended fracture under the alternating fracturing load of casing threads.

To sum up, according to the stress analysis of the long round thread joint under different state of $\mathrm{W}$ well casing, the casing thread joint is damaged due to the impact of drilling measures and complex borehole trajectories in horizontal Wells. In the fracturing process, due to the combined effect of alternating loads, the casing breaks eventually.

\section{Conclusion}

(1) The stress distribution of the teeth is reasonable, the interference displacement of the teeth is linear, and the contact force of the common buckle is evenly distributed.

(2) During the process of casing lowering, the maximum stress of casing thread appears at the bottom of the 5- button tooth at the big end of the common buckle. When the yield strength is exceeded, a dangerous interface and damage occurs in the plasticity, and the casing begins to crack and extend under the alternating fracturing load of the casing thread. The finite element simulation is consistent with the actual situation.

(3) Through the finite element method, the solid model of $51 / 2$ " API casing long round thread is established, stress of screw joint under different working conditions is analyzed. It provides the theoretical basis for the ultimate bearing capacity of casing thread connection in site and has guiding significance for the standard site construction.

\section{References}

1. Zhang, X, Hu, L, et al. Fracture failure analysis of API connection casing $[\mathrm{J}]$. Sichuan Mctallurgy, 2016(3).

2. Zeng, Z, Wang, F Y, et al. Fracture failure analysis of the Thread of the P110 Casing Pipe[J]. Physical Testing and Chemical Analysis-Part A: Physical Testing. 2017(5).

3. Zhang, J, Y. Wang, J D, et al . .Failure Analysis on Casing Dropout of $\Phi 139.7 \times 12.7 \mathrm{~mm}$ Q125 Premium Connection[J]. Petroleum Tubular Goods \& Instruments 2016(2) : 47-52.

4. Duan, J , L , Zhu , X, H , X et al . FEM Analysis on a $139.7 \mathrm{~mm}$ Buttress Casing Thread[J]. Oil Field Equipment. 2008(11): 64-67.

5. Lian, Z, H, Mou, Y, S, et al. Analysis and control measures on the air tightness detecting pressure of tubing in ultra-deep gas wells[J]. Oil Drilling \& Production Technology. 2018(3): 324-329.

6. Lian, Z, H , Liu , Y. et al. Failure Analysis of 4 1/2 REG turbine shaft connection thread under complex working conditions[J]. Petroleum Drilling Techniques . 2018,46(03): 53-58.

7. Liu , Y, Cui , J, W, et al. Finite element analysis of premium connection under combined load[J]. Machine Design and Manufacturing Engineering, 2017(8).

8. Chen, R, H, Cui , J, W, et al. Failure Analysis of the Fatigue Testing Clamp Thread Based on ABAQUS[J]. Research and Exploration in Laboratory, 2015, 34(11):22-25.

9. Zhang, L, Wang, J, J , et al. Finite element analysis of premium thread of conical-spherical seal structure[J]. Petrochemical Industry Application 2019, 38(05): 55-59.

10. Xu , H , L. Study on joint strength and sealing theory for casing and tubing premium connections[D]. 2015. Southwest Petroleum University. 